\title{
Stellar Populations at Higher Redshifts
}

\section{Tadayuki Kodama}

National Astronomical Obseravtory of Japan, 2-21-1 Osawa, Mitaka, Tokyo 181-8588, Japan email: kodama@optik.mtk.nao.ac.jp

\begin{abstract}
We will review some recent advances in understanding the evolution of stellar populations in galaxies at relatively higher redshifts $(z \gtrsim 0.5)$. We will focus on "mass assembly", "down-sizing" and "high- $z$ zoo" which have been among the most major topics and have seen great observational achievements in the past few years. In particular, wide-field near-infrared surveys, large spectroscopic surveys, and the Spitzer observations at infrared wavelengths, have unveiled underlying stellar masses and dusty star formation in high-z galaxies, which have been crucial in making such recent progresses.
\end{abstract}

Keywords. galaxies: evolution, galaxies: formation, galaxies: luminosity function, mass function, galaxies: stellar content, galaxies: high-redshift, galaxies: clusters: general, cosmology: largescale structure of universe

\section{Introduction}

The current theoretical picture of structure formation in the Universe is the bottom-up growth of large systems by assembly of many building blocks. Figs. 1 show theoretical predictions for cluster formation on the left and galaxy formation on the right. Cluster scale assembly can be directly observed by wide-field imaging of distant clusters (eg., PISCES, Kodama et al. 2005). We can identify lots of filaments and clumps in and around distant clusters as predicted by numerical simulations. However, when it comes to galaxies where baryon physics come into play, the fundamental picture of hierarchical formation has not been fully tested yet.

We will concentrate on the following three major issues on galaxy formation and evolution from observers point of view.

(a) When are the massive galaxies assembled? Is it consistent with hierarchical models?

(b) What is the origin of down-sizing? Is it consistent with the bottom-up picture?

(c) What is the sampling bias in high-z galaxies? How can we overcome this problem?

\section{Mass assembly}

The first important issue is the epoch of assembly of massive galaxies, as it directly tests the hierarchical picture of galaxy formation. In the hierarchical models, stellar mass function (SMF) of galaxies is expected to change dramatically with redshift (e.g. Kauffmann \& Charlot 1998), because massive galaxies are end products of successive mergers of building blocks and therefore they tend to be assembled late in the relatively recent past. Do we really see this change in SMF in the real Universe?

There have been tremendous efforts put in this critical test, and in fact, many widefield near-infrared (NIR) surveys (e.g., K20 (52 $\left.\operatorname{arcmin}^{2}\right)$; GOODS-MUSIC (160 $\left.\operatorname{arcmin}^{2}\right)$; UKIDSS $\left(0.6 \mathrm{deg}^{2}\right)$ ) were conducted and have quantified the evolution in SMFs (or Kband luminosity functions, hereafter KLFs) as a function of redshift. These wide-field surveys demonstrate that there is no significant evolution at the massive end of SMFs or the amount of evolution is consistent with pure passive evolution at the bright end of 

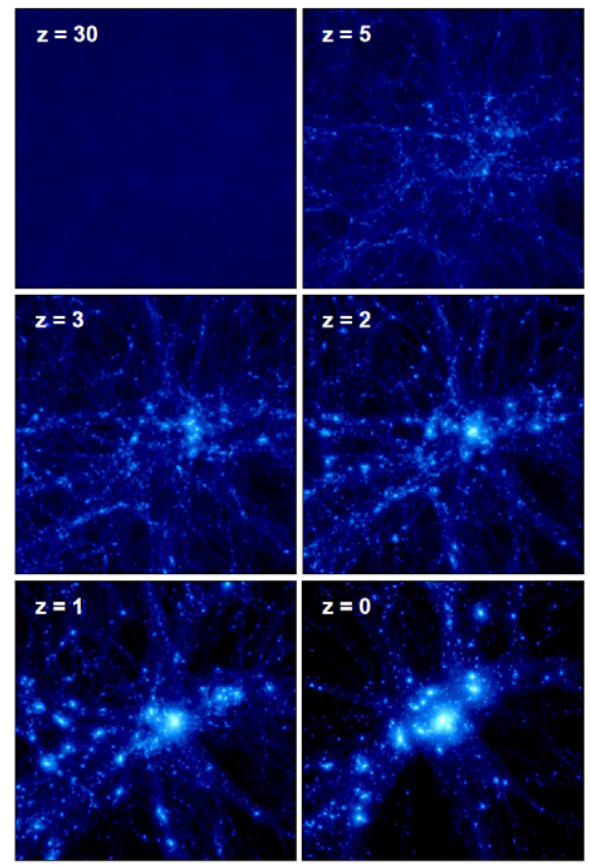

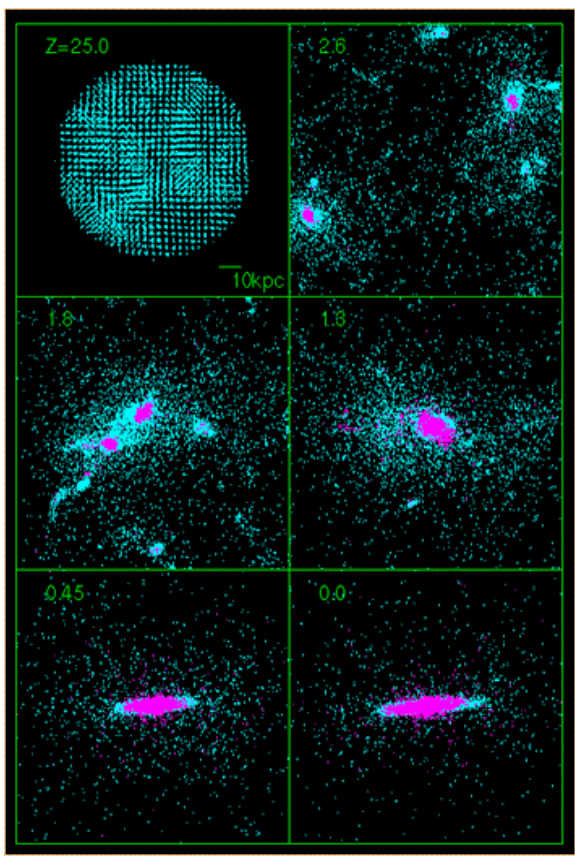

Figure 1. left panel: Dark matter evolution on a cluster scale predicted by $\nu \mathrm{GC}$ simulation by Nagashima et al. (2005). Mass of the cluster is $6 \times 10^{14} \mathrm{M}_{\odot}$ at $z=0$. right panel: A SPH simulation of a Milky-Way type galaxy formation by Bekki \& Chiba (2001).

KLFs up to redshift of 1-1.5 (e.g. Pozzetti et al. 2003; Drory et al. 2005; Fontana et al. 2006; Cirasuolo et al. 2006). Combined with the fact that SED of massive galaxies evolve passively in general at $z<1$ (Section 3) hence the assumption of passive evolution is justified independently, it can be concluded that the majority of the massive galaxies seen today had already been assembled by $z=1-1.5$, namely the first $1 / 2-1 / 3$ of the age of the present-day Universe. There is a caution, however, that the brightest cluster galaxies (such as $\mathrm{cD}$ ) sitting in the deepest potential wells of massive clusters may have a different story and they may be still growing by further accretion/mergers even at $z<1$ (Aragón-Salamanca et al. 1998). We should also note that some studies (e.g., van Dokkum 2005; Bell et al. 2004; Faber et al. 2005) claim much later assembly of massive galaxies by major mergers of gas poor systems ('dry merger') at $z<1$.

What about higher redshifts? Beyond $z=2$, an efficient way of tracing stellar mass is to search for distant red galaxies (DRGs, Franx et al. 2003), with a single colour cut of $J-K>2.3$. Such surveys have been conducted intensively (e.g., van Dokkum et al. 2003; 2004; 2006; Förster Schreiber et al. 2004; Papovich et al 2006), and what they found is that there are a large number of very massive galaxies, more massive than $10^{11} \mathrm{M}_{\odot}$ in stellar mass, and that DRGs host almost $80 \%$ of the entire stellar mass at $2<z<3$. However, the co-moving number density of such massive galaxies starts to decrease with redshift beyond $z=1.5$ or so, as is shown in some recent deep surveys at NIR and Spitzer bands (Drory et al. 2005; Fontana et al. 2006). Decline in the number of massive galaxies is also reported in proto-clusters (Kodama et al. 2007). As shown in Fig. 3, the massive end $\left(\mathrm{M}_{\text {stars }}>10^{11} \mathrm{M}_{\odot}\right)$ of the red sequence of galaxies in proto-clusters seems to grow rapidly between $z=3$ and 2 . These are indirect pieces of evidence that massive galaxies start to break down into building blocks and are yet at the stage of assembly 


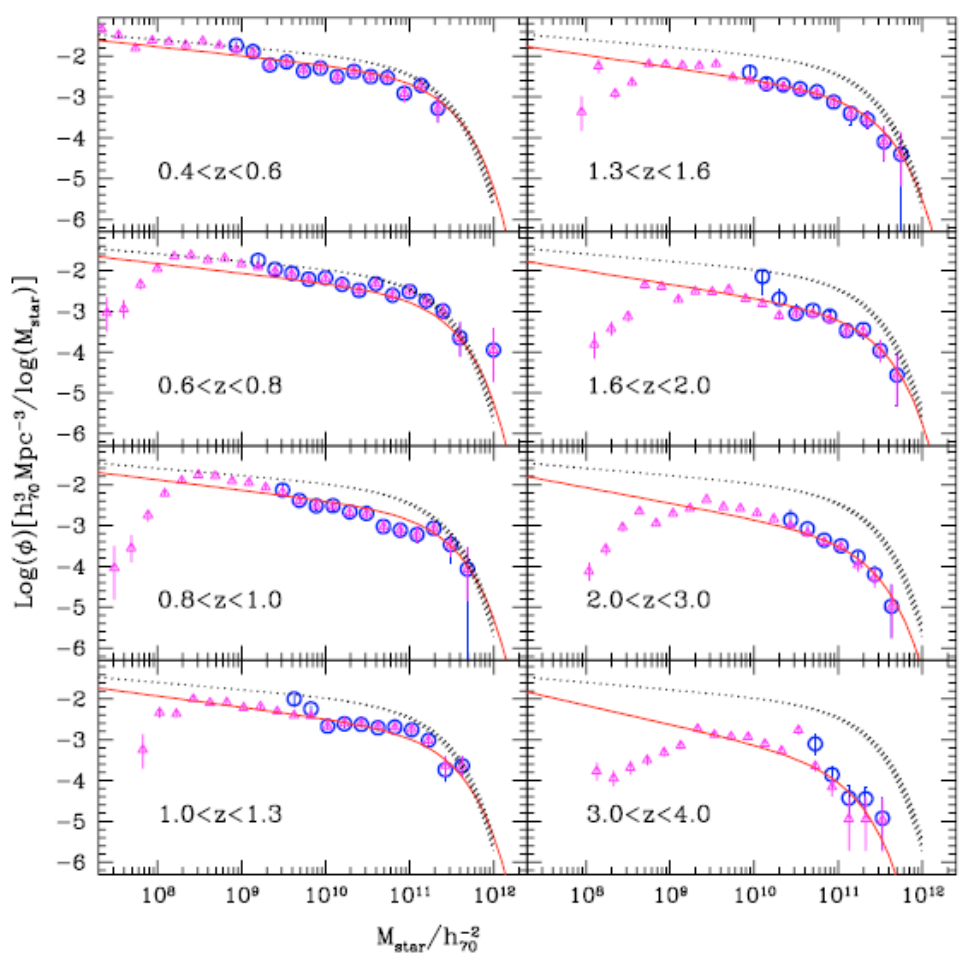

Figure 2. Evolution of stellar mass function with redshift derived from the GOODS-MUSIC survey (Fontana et al. 2006).

at $z>1.5$. Some direct pieces of evidence of mass assembly or mergers in action are also reported in high resolution imaging of distant galaxies. For example, the central radio galaxy in a proto-cluster at $z \sim 2.16$ turns out to be consisting of many building blocks by HST/ACS imaging (Miley et al. 2006). It is interesting to note such an era of rapid growth of massive galaxies coincides with the peak of the submm phase and the peak of the cosmic star formation rate (e.g., Chapman et al. 2005; Bouwens et al. 2005). Therefore, massive galaxies are just forming during this epoch by vigorous star formation and assembly.

Such relatively early formation of massive galaxies $(z>1.5)$, however, can be a serious problem for the hierarchical galaxy formation models. Cimatti et al. (2005) showed that the fraction of massive galaxies in a semi-analytic model (De Lucia et al. 2006) decreases much faster than what is observed even below redshift of unity. However, some other recent models seem to do a better job, not only at $z \sim 1$ but even at higher redshifts. Fontana et al. (2006) compared the observed SMFs with various model predictions. There are at least two models which can reproduce the observed stellar mass density and the SMFs at high redshifts. One is the new Durham semi-analytic model (Bower et al. 2006) which has now taken AGN feedback into account. And the other is the hydro-dynamical simulation by Nagamine et al. (2005). Both models have succeeded in reproducing enough number of massive galaxies at high redshifts. 

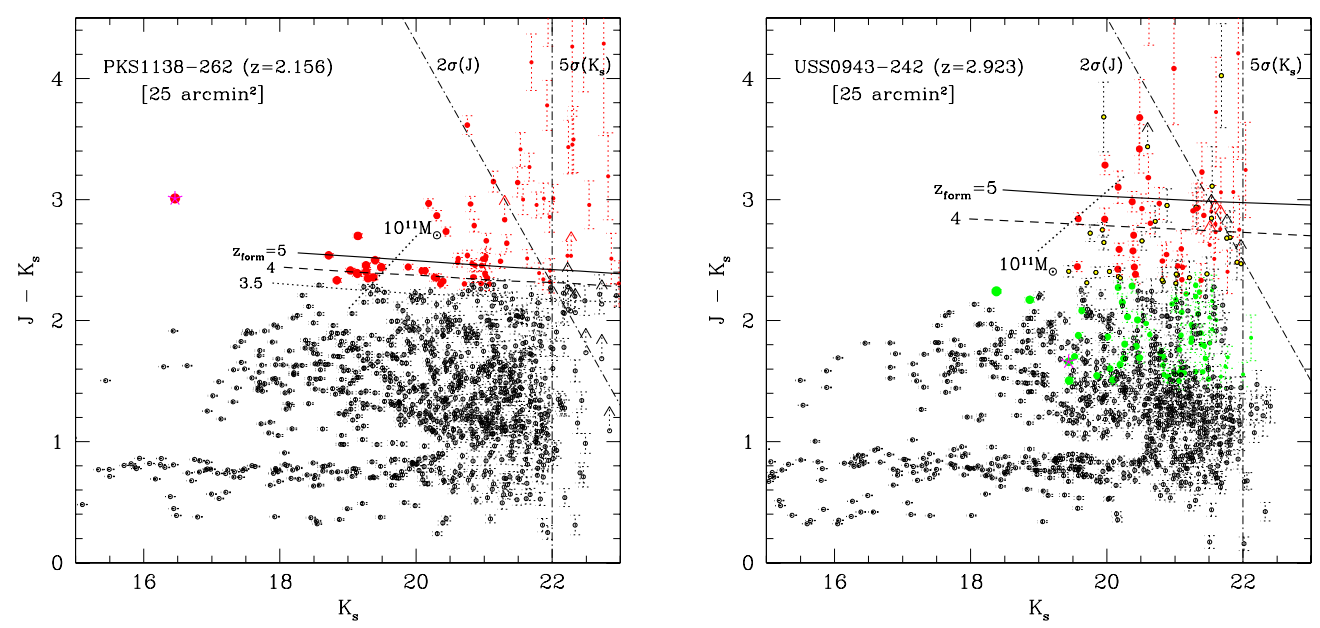

Figure 3. Near-infrared colour-magnitude diagrams of proto-clusters at $z \sim 2$ (left) and at $z \sim 3$ (right) (Kodama et al. 2007). The predicted location of the colour-magnitude sequence is shown in the case of passive evolution with various formation redshifts (Kodama et al. 1998). The iso-stellar mass lines at $10^{11} \mathrm{M}_{\odot}$ are also shown. See Kodama et al. (2007) for further details.

\section{Down-sizing}

Let us now turn to less massive galaxies. It was first noted by Cowie et al. (1996) that massive galaxies are the oldest and less massive galaxies tend to be younger or have extended star formation. They named this phenomenon "down-sizing" as star formation activities propagate from massive systems to less massive systems as time progresses. Recent surveys have confirmed that this trend extends to much fainter magnitudes. Kauffmann et al. (2003) showed using the SDSS data that massive galaxies are red and old while less massive galaxies tend to be blue and younger. This trend is also seen at higher redshifts. Galaxies at $z \sim 1$ show a very similar bimodal distribution on the colourmagnitude diagram (Kodama et al. 2004). Massive end is almost completely dominated by red old galaxies while the low mass end is exclusively dominated by blue galaxies and the transition occurs at around a few times $10^{10} \mathrm{M}_{\odot}$ in stellar mass.

There have been many papers since then which confirms the down-sizing picture from various aspects. These includes a fundamental plane analysis of early-type galaxies at $z \sim 1$ (e.g., Treu et al. 2005; van der Wel et al. 2005), chemical enrichment of gas in HII regions at $z \sim 2$ (e.g., Erb et al. 2006), and Mg/Fe ratio of local early-type galaxies (e.g., Thomas et al. 2002). All these results indicate clearly that less massive galaxies have had more extended star formation history compared to massive, older galaxies. The most convincing case has recently come from Bundy et al. (2006) based on 8,000 galaxies with spectroscopic redshifts between $0.4<z<1.4$ taken by the DEEP2 survey (Davis et al. 2001). As reproduced in Fig. 4, SMFs of red and blue galaxies are shown separately as a function of redshift. They find again that massive end is dominated by red galaxies, while low mass end is dominated by blue galaxies at all redshifts. What is interesting here is that the critical mass that separates the red and the blue populations actually shifts to lower mass as we go to lower redshift as illustrated by the arrow. This is the true "down-sizing" seen as a function of time, and star formation is truncated from massive galaxies to low mass galaxies as time progresses.

What if the down-sizing is now seen as a function of environment? Based on the widefield data on cluster outskirts, Tanaka et al. (2005) has shown similar diagrams but 


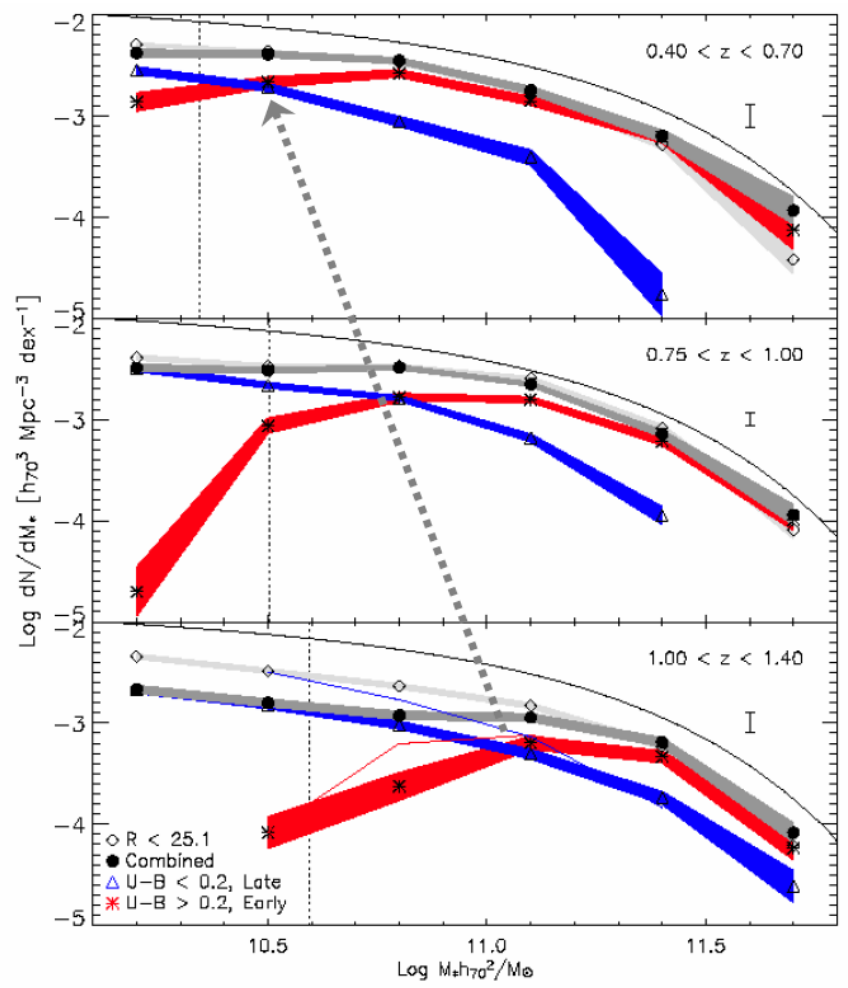

Figure 4. "Down-sizing" evolution in star formation of galaxies from the DEEP2 spectroscopic survey (Bundy et al. 2006). Red, Blue, and grey curves are stellar mass functions of red, blue and total galaxies, respectively, as indicated in the bottom panel. An arrow indicates how the dividing mass between red and blue populations shifts to lower masses as time progresses.

are now divided into three environmental bins, namely, field, groups and cluster cores (Fig. 5). The red solid and blue dashed curves indicate luminosity functions of the red and the blue galaxies, respectively. The critical mass that separates the red and the blue populations now shifts to lower mass as we go to higher density regions. This is similar to what we see above as a function of redshift. Therefore, the galaxy environment also controls the speed of galaxy evolution, and star formation propagates from high density regions to lower density regions.

Combining the above results based on Figs. 4 and 5, the star formation history of galaxies can be summarised as follows: Star formation is truncated from high-mass systems in high density regions to low-mass systems in low density regions.

Critical question on the down-sizing picture is that how it can be accommodated in the hierarchical galaxy formation scenario which is apparently opposite, namely, early formation of small systems and later formation of massive galaxies would have been expected. However, recent semi-analytic models do reproduce the down-sizing in star formation at least in a qualitative sense (De Lucia et al. 2006). In these models, massive galaxies tend to have star formation earlier than less massive ones in consistent with the observations. This is probably because of a strong spatial bias in galaxy formation, in the sense that massive galaxies were formed from higher density peaks at high redshifts which collapsed earlier and had accelerated galaxy formation, while less massive galaxies started from lower density peaks and had delayed formation. There is such an intrinsic 
SDSS $(\mathrm{z} \sim 0)$

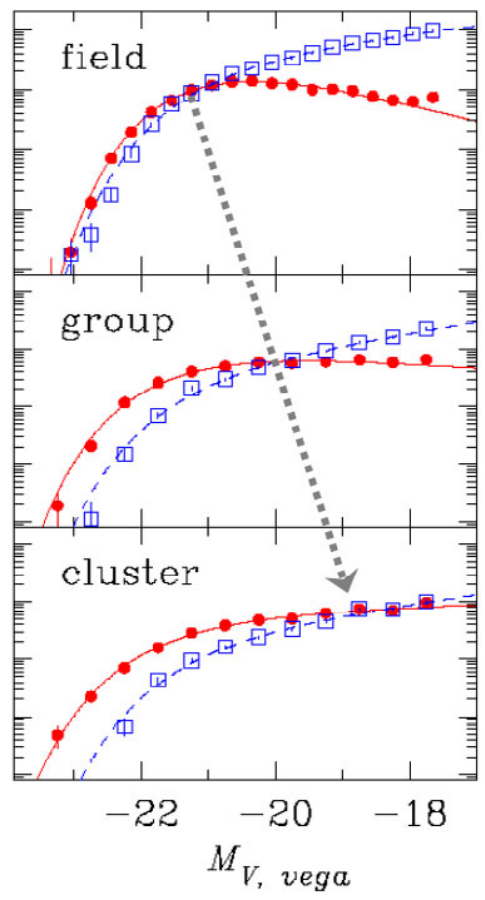

RXJ0152 ( $\mathrm{z}=0.83)$

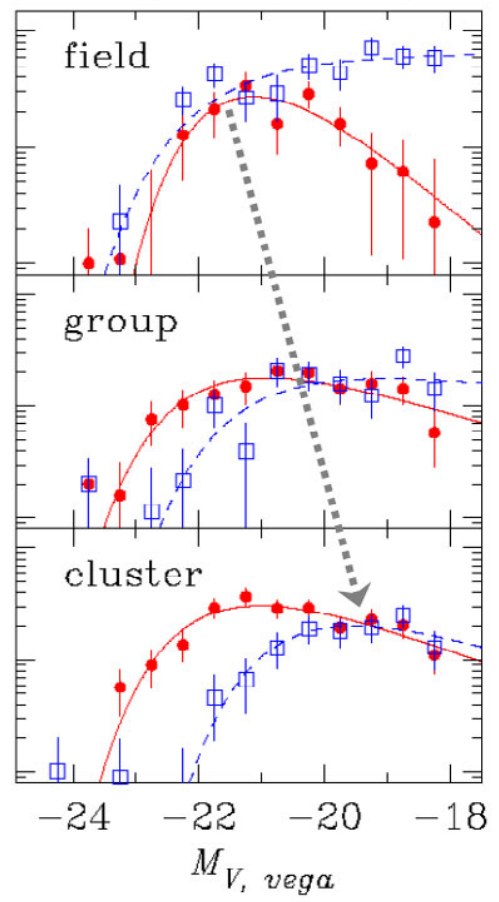

Figure 5. "Down-sizing" seen as a function of environment (Tanaka et al. 2005). Filled circles and blue squares show red and blue populations, respectively. Two arrows indicate how the dividing mass between red and blue populations shifts to lower mass as one goes to denser environments.

bias which can inverse the bottom-up picture. But even so, it is not clear how we can extend star formation in such small systems where SN feedback can easily expel the gas. Extended star formation in these low mass systems would require extremely low star formation efficiency or recurrent star formation by the fall back of once expelled gas.

\section{High-z zoo}

High- $z$ Universe is completely a mess and it is called high- $z$ zoo. There are a wide variety of galaxy populations with three characters, such as LAE, LBG, SMG, DRG, BzK, JHK and so on. Selections of each population are all different and so each sample is biased in some way, and we do not know the evolutionary state of each population nor the evolutionary link between different populations. This situation makes it extremely difficult for us to obtain any general view of high- $z$ galaxies. In order to better understand the galaxy formation, what is needed is to place all these different populations on a single diagram with the common fundamental physical quantities. One of such key diagrams is shown in Fig. 6. We should first construct a stellar mass limited sample, and then measure specific star formation rate (SSFR) of each galaxy. SSFR is the current SFR per unit stellar mass and is very similar to the Kennicutt's birth parameter (1998) and is also directly connected to gas fraction through the Schmidt law (Schmidt 1959; Tinsley 1980). It is therefore a good measure of evolutionary state of a galaxy.

One of the major uncertainties in making such diagram at high redshifts is the effect of dust extinction on measuring true star formation rates (SFRs). In fact star formation 

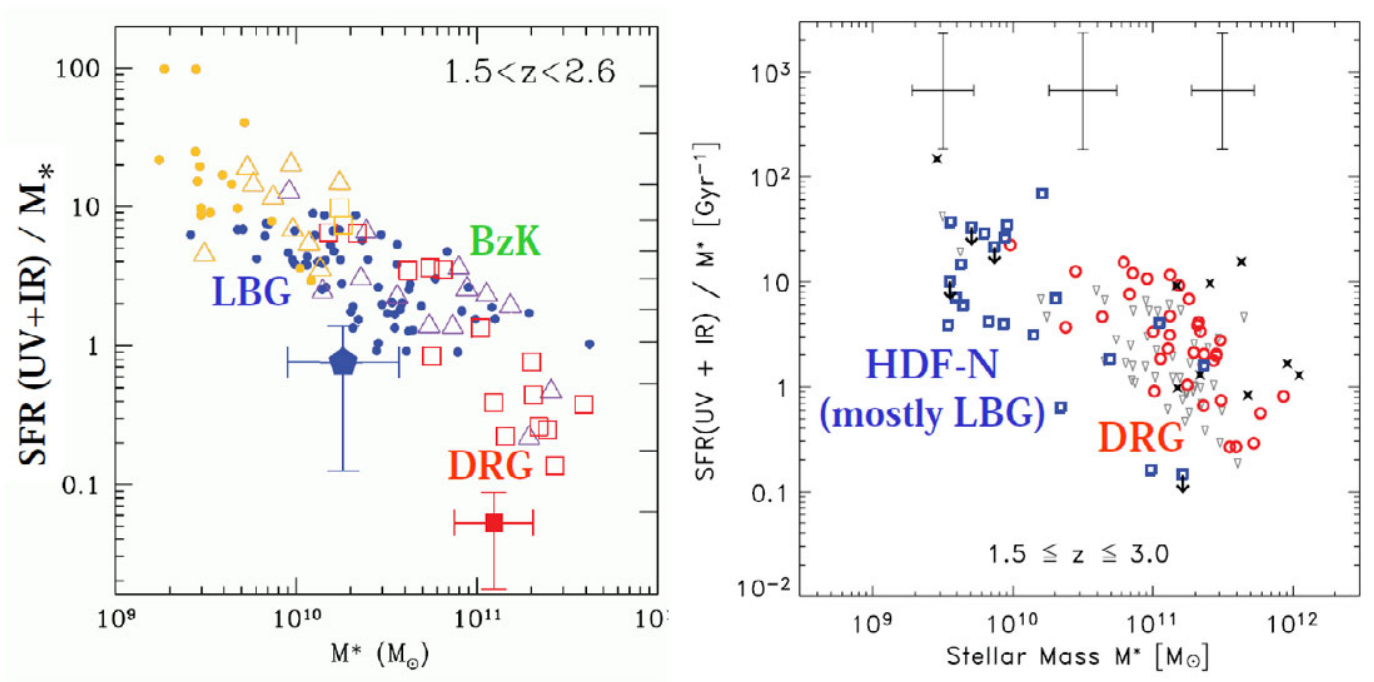

Figure 6. left panel: Specific star formation rate (SSFR) as a function of stellar mass of galaxies (Reddy et al. 2006). Star formation rates (SFRs) are measured as sums of UV-SFR and infrared-SFR. right panel: Similar diagram but from Papovich et al. (2006). Different symbols indicate different populations.

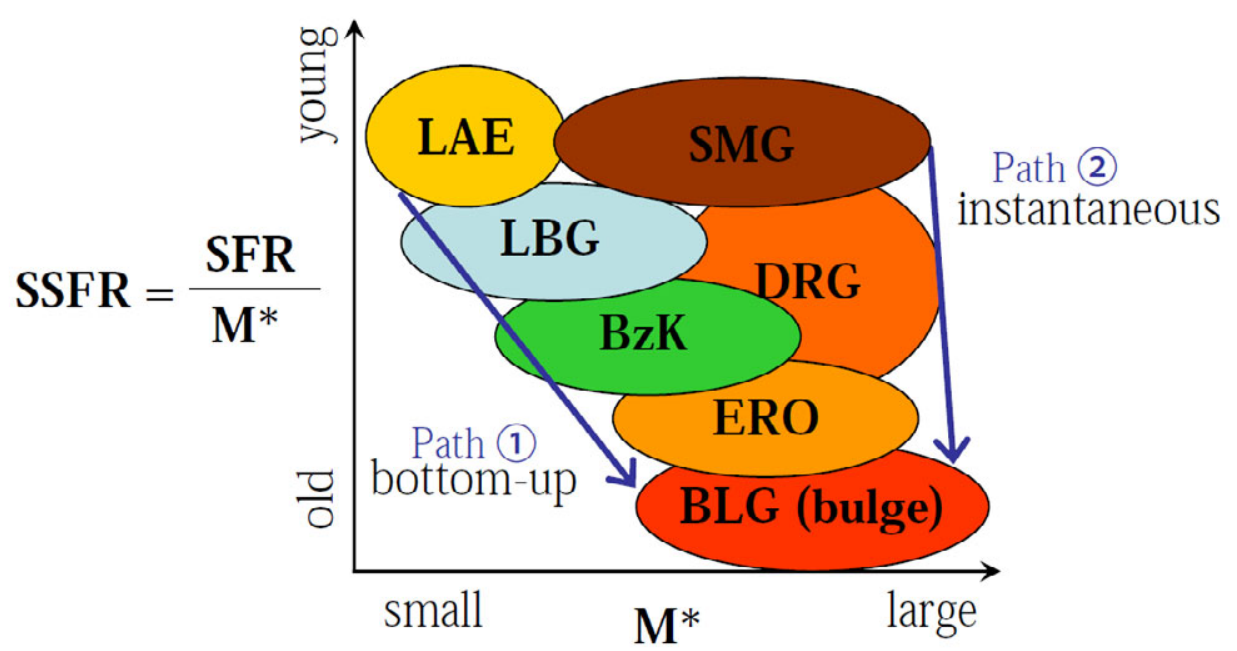

Figure 7. Schematic diagram of the "High- $z$ zoo", where a wide variety of 3-character populations have been defined. Neither an evolutionary connection nor inter-relationships are understood well. This diagram sketches the author's personal view of high- $z$ populations on this key diagram showing the specific star formation rates (SSFR) versus stellar masses. Therefore it should be taken carefully. Two arrows indicate two possible paths to form a bulge dominated massive galaxies in the authors view.

is largely hidden in optical surveys at high redshifts, and correction for dust extinction is critically important to derive the cosmic star formation history, for example (e.g., Bouwens et al. 2005) Spitzer Telescope observations play a key role here by providing unique information between $3 \mu \mathrm{m}$ and $24 \mu \mathrm{m}$. First of all, the Spitzer bands are essential to accurately estimate stellar masses and photometric redshifts at high- $z$ by SED fitting. 
In fact, without the Spitzer data, mass estimates would become very uncertain beyond $z \sim 2$ or so (Fontana et al. 2006). Moreover, MIPS 24micron samples PAH features at rest frame $6-9 \mu \mathrm{m}$ from $z \sim 2$ galaxies which are good measures of dusty star formation rates. Webb et al. (2006) actually measured the star formation rates for the MIPS detected DRGs, and found that their average star formation rate is as high as $130 \mathrm{M}_{\odot} / \mathrm{yr}$, and that DRGs contribute $20 \%$ of the total SFR density at $z \sim 2$. Furthermore, Spitzer colours can be used to discriminate among passively evolving galaxies, dusty red galaxies, and the galaxies with a significant AGN component (e.g., Wilson et al. 2006; Stern et al. 2005; Webb et al. 2006).

Fig. 6 show the two pioneer works which combine UV star formation and IR star formation derived from the Spitzer data, and they created the ultimate diagrams at $z \sim 2$. Down-sizing is again clearly reproduced on this key diagram even at this high redshift. It is also clear that $\mathrm{LBG}$ and DRG are well separated on this diagram, in the sense that DRGs are more massive and more evolved systems than LBGs.

Although it is still too early to show it, we try to summarize the high- $z$ zoo on the schematic diagram shown in Fig. 7. There are apparently two paths to form a massive spheroidal galaxy. One is the gradual bottom-up starting from LAE, evolving to LBG and BzK, and eventually to a bulge dominated massive galaxy. The other path would be an instantaneous early formation of massive galaxy. A galaxy is formed by a strong star burst in a short timescale and is seen as submm galaxies (SMG) during the burst phase. By increasing the sample of galaxies on this ultimate diagram in the near future, and plotting them as a function of redshift, we can eventually make an evolutionary link between different galaxy populations and establish a concrete picture of galaxy formation based purely on observations. That is the way to go.

\section{Acknowledgements}

This work was financially supported in part by a Grant-in-Aid for the Scientific Research (No. 18684004) by the Japanese Ministry of Education, Culture, Sports and Science.

\section{References}

Aragon-Salamanca, A., Baugh, C. M., \& Kauffmann, G. 1998, MNRAS 297, 427

Bekki, K., \& Chiba, M. 2001, ApJ 558, 666

Bell, E. F., et al. 2004, ApJ, 608, 752

Bouwens, R. J., Illingworth, G. D., Thompson, R. I., \& Franx, M. 2005, ApJ 624, L5

Bower, R. G., Benson, A. J., Malbon, R., Helly, J. C., Frenk, C. S., Baugh, C. M., Cole, S., \& Lacey, C. G. 2006, MNRAS 370, 645

Bundy, K., et al. 2006, ApJ 651, 120

Chapman, S. C., Blain, A. W., Smail, I, \& Ivison, R. J. 2005, ApJ 622, 772

Cimatti, A., Daddi, E., \& Renzini, A. 2006, A\&A 453, L29

Cirasuolo, M., et al. 2006, astro-ph/0609287

Cowie, L. L., Songaila, A., Hu, E. M., \& Cohen, J. G. 1996, AJ 112, 839

Davis, M., Newman, J. A., Faber, S. M., \& Phillips, A. C. 2001, Deep Fields, 241

De Lucia, G., Springel, V., White, S. D. M., Croton, D., \& Kauffmann, G. 2006, MNRAS 366, 499

Drory, N., Salvato, M., Gabasch, A., Bender, R., Hopp, U., Feulner, G., \& Pannella, M. 2005, ApJ 619, L131

Erb, D. K., Shapley, A. E., Pettini, M., Steidel, C. C., Reddy, N. A., \& Adelberger, K. L. 2006, ApJ 644, 813

Faber, S. M. 2005, astro-ph/0506044

Fontana, A., et al. 2006, A\& $A$ 459, 745 
Förster Schreiber, N. M., et al. 2004, ApJ 616, 40

Franx, M., et al. 2003, ApJ 587, L79

Kauffmann, G., \& Charlot, C. 1998, MNRAS 297, 23

Kauffmann, G., et al. 2003, MNRAS 341, 54

Kennicutt, R. C. 1998, ARA\&A, 36, 189

Kodama, T., Arimoto, N. Barger, A.J. \& Aragón-Salamanca, A. 1998, A\&\&A 334, 99

Kodama T., et al. 2004, MNRAS 350, 1005

Kodama, T., et al. 2005, PASJ 57, 309

Kodama, T., Tanaka, I., Kajisawa, M., Kurk, L., Venemans, B., De Breuck, C., Vernet, J., \& Lidman, C. 2007, MNRAS submitted

Miley, G. K., et al. 2006, ApJ 650, L29

Nagamine, K., Cen, R., Hernquist, L., Ostriker, J. P., \& Springel, V. 2005, ApJ 627, 608

Nagashima, M., Yahagi, H., Enoki, M., Yoshii, Y., \& Gouda, N. 2005, ApJ 634, 26

Papovich, C., et al. 2006, ApJ 640, 92

Pozzetti, L., et al. 2003, A\&A, 402, 837

Reddy, N. A., Steidel, C. C., Fadda, D., Yan, L., Pettini, M., Shapley, A. E., Erb, D. K., \& Adelberger, K. L. 2006, ApJ 644, 792

Schmidt, M. 1959, ApJ 129, 243

Stern, D., Chary, R.-R., Eisenhardt, P. R. M., \& Moustakas, L. A. 2006, AJ 132, 1405

Tanaka, M., Kodama, T., Arimoto, N., Okamura, S., Umetsu, K., Shimasaku, K., Tanaka, I., \& Yamada, T. 2005, MNRAS, 362, 268

Thomas, D., Maraston, C., \& Bender, R. 2002, Ap\&SS 281, 371

Tinsley B. M. 1980, Fundamentals of Cosmic Physics Vol.5, p.287

Treu, T., Ellis, R. S., Liao, T. X., \& van Dokkum, P. G. 2005, ApJ 622, L5

van der Wel, A., Franx, M., van Dokkum, P. G., Rix, H.-W., Illingworth, G. D., \& Rosati, P. 2005, ApJ 631, 145

van Dokkum, P. G. 2005, $A J$ 130, 2647

van Dokkum, P. G., et al. 2003, ApJ 587, L83

van Dokkum, P. G., et al. 2004, ApJ 611, 703

van Dokkum, P. G., et al. 2006, ApJ 638, L59

Webb, T. M. A., et al. 2006, ApJ 636, L17

Wilson, G., et al. 2006, astro-ph/0608447

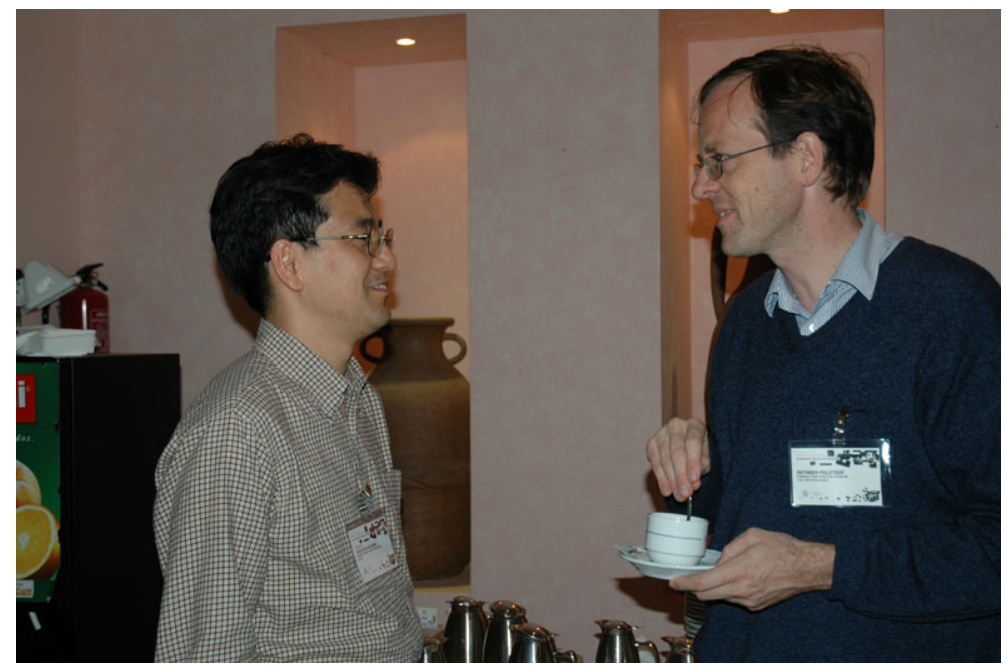

The speaker (left) talking to Reynier Peletier. 